\title{
Presença da Proteina p53 como Prognóstico de Recidiva/Progressão de Neoplasia Intra-epitelial Vulvar III
}

\author{
p53 Protein Overexpression as a Prognostic Marker for Vulvar Intraepithelial \\ Neoplasia III Recurrence/Progression

\begin{abstract}
Isabel Cristina Chulvis do Val Guimarães ${ }^{1}$, Gutemberg Leão de Almeida Filho ${ }^{2}$, Maria da Glória de Carvalho ${ }^{3}$, Christina Maeda Takiya ${ }^{4}$, Aldo Franklin Ferreira Reis ${ }^{5}$, Paulo Marcos Valiante ${ }^{6}$, Maria Consuelo Gondim ${ }^{7}$
\end{abstract}

\section{RESUMO}

Objetivo: avaliar o valor da presença da proteina p53 nos casos de recidiva/progressão da neoplasia intra-epitelial vulvar (VIN) III.

Métodos: foram selecionadas 20 pacientes com VIN III indiferenciada, seguidas semestralmente por periodo de até quatro anos, divididas em dois grupos: quatorze sem e seis com recidiva/ progressão da lesão. Os casos de recidiva/ progressão foram distribuidos da seguinte forma: em três pacientes a recidiva ocorreu uma única vez, em duas, houve dupla recorrência e apenas uma evoluiu para carcinoma escamoso. Em ambos os grupos foram avaliados o sítio vulvar acometido e a presença da proteina $p 53$ com análise do padrão de marcação imunohistoquimica. Estudo semelhante foi realizado nos casos de recidiva/ progressão além da análise do intervalo de tempo para o surgimento de recidiva/ progressão. Resultados: observou-se recidiva da VIN III em 25\% dos casos e, em 5\%, progressão para carcinoma. O tempo médio de recidiva foi de 24,5 meses. A localização multifocal da lesão primária foi a mais freqüente (50\%) em ambos os grupos. Na maioria dos casos (87,5\%), a recidiva/progressão ocorreu na mesma localização da lesão vulvar primária. A presença da proteina p53 mostrou-se positiva em 50\% das lesões primárias de VIN III e em $75 \%$ dos casos de recidiva/progressão.

Conclusões: a presença da proteína p53 parece desempenhar papel importante na gênese e na predição do curso clínico das VIN III. As recidivas/progressão das VIN III tendem a ocorrer na mesma área da doença inicial, sugerindo a presença de campo molecular alterado.

PALAVRAS-CHAVE: Neoplasia intra-epitelial vulvar. Vulva, lesões pré-neoplásicas. Oncogenes.

\section{Introdução}

As neoplasias intra-epiteliais vulvares (VIN) apresentam percentual de recidiva entre 10 e $40 \%$ contra cerca de $5 \%$ de casos que evoluem para carcinoma invasor ${ }^{1}$. É evidente que o pequeno grupo de mulheres mais idosas com história de múl-

Instituto de Ginecologia ${ }^{1,2,7}$, Laboratório Multidisciplinar de Pesquisa e de Microscopia Eletrônica ${ }^{4,6}$, Laboratório de Controle de Expressão Gênica da Universidade Federal do Rio de Janeiro ${ }^{3}$ e Faculdade de Medicina de Campos $^{5}$ Correspondência:

Isabel Cristina Chulvis do Val Guimarães

Rua General Roca, 935/904 - Tijuca

20521-070 - Rio de Janeiro - RJ

Tel.: (21)22547790

e-mail: isabeldoval@openlink.com.br tiplas neoplasias intra-epiteliais do trato genital inferior, que progrediram para câncer de vulva em estudos anteriores, já não reflete a situação da VIN III nos dias de hoje ${ }^{1}$. O notável aumento na incidência desta doença nos últimos anos tem nos permitido maior conhecimento sobre ela, tanto em relação à propedêutica quanto ao tratamento e seguimento.

Atualmente, os estudos de biologia molecular têm demonstrado que a simples presença do DNA-HPV não é capaz de determinar a progressão da VIN III para carcinoma invasor ${ }^{2}$. Entretanto, estes ensaios sugerem que alterações genéticas, associadas ou não à infecção induzida por HPV, que ocorrem durante o processo de 
carcinogênese vulvar, seriam pré-requisitos para o desenvolvimento do câncer de vulva ${ }^{3}$.

Estudos genéticos têm identificado diversos genes envolvidos no processo da carcinogênese humana, principalmente aqueles reguladores da entrada da célula no ciclo celular. Eles são subdivididos em duas classes: genes proliferativos ou proto-oncogenes e genes antiproliferativos ou supressores de tumor. Os proto-oncogenes são aqueles que promovem a proliferação celular exercendo, dessa forma, controle positivo sobre o ciclo celular ${ }^{4}$. Entretanto, quando mutados passam a chamar-se oncogenes. Já os genes supressores de tumor, por não estimularem a proliferação celular, exercem controle negativo sobre o ciclo celular. Esses genes podem perder sua função por mutação, por deleção de cromossomos ou por inativação por meio da formação de complexos com proteínas virais ${ }^{5}$.

O gene supressor de tumor P53 desempenha papel fundamental nos processos de crescimento e morte celular. A proteína p53 foi denominada "a guardiã do genoma" devido à sua capacidade de prevenir o acúmulo de alterações genéticas. Essa tarefa é efetuada mediante a parada do ciclo celular com a finalidade de evitar a replicação de DNA danificado e conseqüente proliferação celular desordenada. Outro mecanismo de supressão do desenvolvimento tumoral está ligado à habilidade da p53 em eliminar células aberrantes por iniciar o processo de apoptose ou morte celular programada 4 .

A presença de mutação no gene $P 53$ desencadeia aumento da oncoproteína p53, que é mais estável do que o tipo selvagem encontrado nas células normais. Esta estabilidade se deve à maior meia-vida de proteínas de conformação alterada, as quais acumulam-se no núcleo das células neoplásicas, podendo ser detectadas por métodos imuno-histoquímicos ${ }^{6}$.

No que diz respeito à VIN III, já existem estudos bem fundamentados em relação à sua epidemiologia, aos fatores de risco, aos aspectos clínicos, à histopatologia e ao tratamento. Porém, o mesmo não ocorre no que se refere à recidiva e à progressão.

Acredita-se que alguns fatores possam ser responsabilizados pelas recorrências ou pela progressão das VIN III, tais como a presença do papilomavirus humano (HPV) e o caráter multifocal das lesões. Da mesma forma, os tratamentos destrutivos baseados apenas em resultados de biopsia, limitando a exclusão de lesão invasora; a grande variedade de modalidades terapêuticas; o comprometimento de margens cirúrgicas e de anexos da pele (folículo piloso) parecem contribuir, também, para os casos de recidiva ${ }^{1,7-9}$. Todavia, a literatura ainda é escassa em relação ao comportamento genético das VIN III.

Portanto, este trabalho tem como objetivo avaliar o valor da presença da proteína p53 nos casos de recidiva e de progressão da VIN III.

\section{Pacientes e Métodos}

Trata-se de estudo tipo coorte prospectivo com análises transversais no qual foram incluídas 20 de 26 pacientes com neoplasia intraepitelial vulvar III do tipo indiferenciado do Ambulatório de Patologia Vulvar do Instituto de Ginecologia da Universidade Federal do Rio de Janeiro, tratadas cirurgicamente (excisão total da lesão) no período de julho de 1996 a julho de 2000. A suspeita clínica foi confirmada por biopsia prévia utilizando o punch de Keyes de 3-5 mm. Em seis casos o estudo não pôde ser realizado devido à indisponibilidade dos blocos.

As pacientes foram seguidas semestralmente, a partir do procedimento cirúrgico, por período de quatro anos. De acordo com os resultados obtidos, as 20 pacientes foram divididas em dois grupos: um grupo composto por 14 pacientes que não cursaram com recidiva e outro com cinco pacientes que experimentaram recorrência e uma que evoluiu para carcinoma escamoso. Os casos com recidiva foram distribuídos da seguinte forma: em três pacientes a recidiva ocorreu uma única vez e, em duas, houve dupla recorrência.

Desta forma, um total de 28 blocos parafinados referentes às biopsias das lesões primárias de 20 pacientes com VIN III, de sete recidivas e de uma progressão para câncer escamoso de vulva fizeram parte de nosso estudo.

Em ambos os grupos foram avaliados a localização das lesões na vulva, o tempo de seguimento de cada paciente e a presença da proteína p53 com análise do padrão de marcação pela imunohistoquímica. Estudo semelhante foi realizado nos casos de recidiva/progressão, além da análise do intervalo de tempo entre o tratamento da lesão inicial e a ocorrência da primeira recidiva/progressão.

Para o estudo da distribuição vulvar da lesão primária e das recidivas/progressão decidiuse dividir a vulva em terço anterior, médio e posterior. A recidiva foi considerada de caráter multifocal quando mais de um dos terços da vulva foi acometido. Baseado nos conceitos de Junge et al. ${ }^{9}$, definiu-se como recidiva, independentemente da localização da lesão inicial, o surgimento de nova lesão em qualquer área do território vulvar após intervalo de tempo maior que três meses. 
O material obtido do espécime cirúrgico foi fixado em formaldeído a $10 \%$ tamponado (formol de Lillie) por 24 horas no máximo, e a seguir desidratado e incluido em parafina. Cortes de $5 \mu \mathrm{m}$ foram obtidos e corados com hematoxilina-eosina. A análise histopatológica foi realizada, separadamente, por dois anatomopatologistas. Em nenhum caso houve divergência, por parte dos patologistas, no diagnóstico das lesões vulvares. Utilizaramse apenas cortes de blocos parafinados que continham área de epitélio com mais de 1.000 células.

Para detecção da proteína nuclear p53 foram utilizados cortes de $5 \mu \mathrm{m}$ de espessura, oriundos dos blocos parafinados usados para o diagnóstico histopatológico. Usou-se o anticorpo monoclonal 126 clone DO- 1 cat (SC Biotechnology, USA) produzido em camundongo. A localização da proteína p53 foi realizada pela técnica $A B C$ - peroxidase utilizando o kit Lisab (Dako, USA). Para a revelação empregou-se diaminobenzidina - DAB (Dab Fast - Sigma, USA), seguida de contracoloração pela hematoxilina. Foram usados cortes histológicos incubados com soro não contendo anticorpo de camundongo como controle negativo das reações. Utilizaram-se como controle positivo cortes de tumor cerebral com marcação positiva para a p53, previamente testados. Os cortes histológicos com expressão negativa para a proteína p53 foram submetidos ao teste da vimentina. Trata-se de teste de controle de reatividade imunocitoquímica verificado por técnica imuno-histoquímica, tendo como finalidade a exclusão dos espécimes cirúrgicos negativos para vimentina, indicando que foram processados indevidamente.

A avaliação da expressão nuclear da proteína p53 foi realizada de forma semi-quantitativa, ou seja, campos celulares contendo em média 1.000 células foram analisados em microscópio de luz, com objetiva de 40X e ocular de 10X, adotando-se os critérios propostos por Emanuels et al. ${ }^{10}$, que valorizam não somente a quantidade de células que expressam a presença da proteína $\mathrm{p} 53$, mas também a intensidade e a distribuição de marcação nuclear da proteína p53 (Figura 1A, B).

Foram considerados positivos os casos nos quais se detectou presença da proteína p53 em número igual ou maior que 100 células para 1.100 negativas (maior ou igual a 10\%).

A intensidade de marcação foi classificada em forte ou fraca. A marcação forte é aquela na qual a coloração marrom vista nos casos positivos é extremamente pronunciada, fato este que não ocorre no padrão de marcação fraca, que se apresenta sob forma mais clara.

O tipo de marcação foi subdividido em difuso e focal. Nos casos com padrão difuso se verifica homogeneidade de marcação, ou seja, quase to- dos os campos apresentam-se com células marcadas pelo monoclonal anti-p53. No tipo focal apenas grupamentos celulares apresentam células positivas para a proteína da p53.
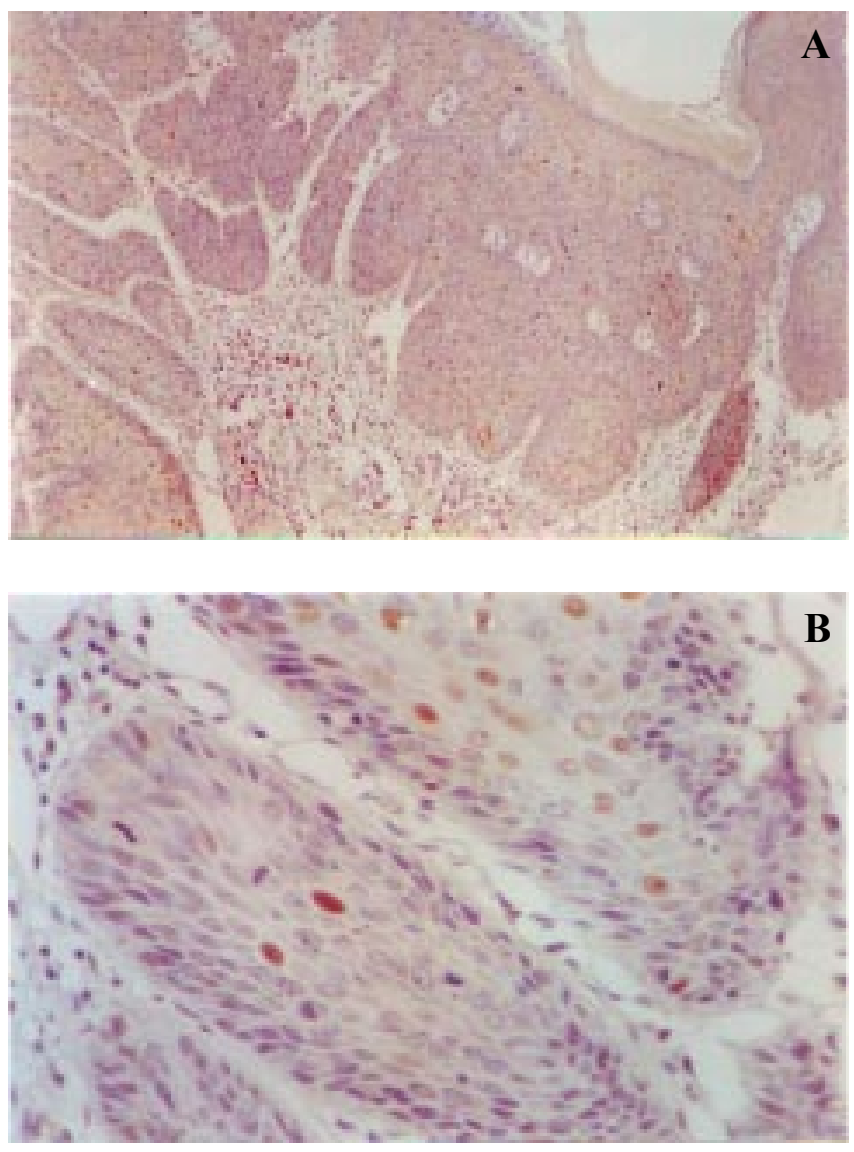

Figura 1 - Lesão primária de VIN III - Reatividade imunohistoquímica para a proteína p53 com padrão de marcação do tipo difuso com intensidade forte (A: X100; B: X400).

A análise estatística foi realizada pelo teste de significância exato de Fisher e do $\chi^{2}$, para comparação entre os dois grupos (sem e com recidiva/progressão) das diferentes variáveis avaliadas. $\mathrm{O}$ critério de determinação de significância foi o nível de $5 \%$, ou seja, para valor de $\mathrm{p} \leq 0,05$ atribuise significância estatística.

Este estudo foi avaliado e aprovado pela Comissão de Ética em Pesquisa do Instituto de Ginecologia da UFRJ e realizado após assinatura do consentimento pós-informado.

\section{Resultados}

Do total de 20 pacientes estudadas, observou-se que uma evoluiu com progressão da lesão para carcinoma escamoso (5\%) e cinco cursaram com recidiva da doença (25\%). Dos cinco casos de recorrência, três pacientes apresentaram um 
único episódio de recidiva, ao passo que duas outras, dois episódios.

Em relação à lesão primária, a localização multifocal foi a mais freqüente $(50 \%)$, tanto no grupo com recidiva (20\%) quanto no sem recidiva (30\%). Quando a doença atingiu apenas um terço vulvar, o posterior foi o mais acometido (30\%), tanto no grupo com recidiva e no único caso de progressão $(10 \%)$, quanto no grupo sem recorrência (20\%). Entretanto, a diferença estatística entre os dois grupos não foi significativa $(\mathrm{p}=0,62)$.

A localização das lesões nos casos com recidiva e no único caso de progressão para carcinoma escamoso foi concordante com a localização da lesão primária em $87,5 \%$ dos casos. Em apenas um caso $(12,5 \%)$ a localização da lesão foi diferente da lesão inicial.

A maioria das recidivas $(66,6 \%)$ aconteceu em período maior que 12 meses. Entretanto, é importante ressaltar que um caso de recidiva e o único de progressão ocorreram em periodo inferior a um ano. O tempo médio para aparecimento de re- cidiva foi de 24,5 meses, com desvio padrão de 19,2 meses, mínimo de 6 e máximo de 48 meses.

Do total de pacientes, $15(75 \%)$ foram seguidas por periodo maior que 12 meses. As cinco mulheres $(25 \%)$ que foram acompanhadas por período inferior a um ano ingressaram em nossa pesquisa sete meses antes de seu término.

A média de tempo do seguimento das 20 pacientes com VIN III foi de 30 meses (amplitude 7 48 meses). Nenhuma paciente do grupo recidiva/progressão foi seguida por menos de 12 meses. Das seis mulheres com recidiva/progressão, cinco foram acompanhadas por período superior a 36 meses.

A presença da proteína p53 foi observada em $50 \%$ das lesões primárias de VIN III. Deste percentual, 30\% corresponderam aos seis casos de recidiva/progressão. Os outros $20 \%$ referem-se às quatro pacientes seguidas por apenas seis meses. Nos casos em que não houve recidiva/progressão da doença não se verificou presença da proteína da p53 (Tabela 1).

Tabela 1 - Presença da proteína p53 na lesão primária de VIN III nos grupos sem e com recidiva/progressão.

\begin{tabular}{lccr}
\hline $\begin{array}{l}\text { Presença da } \\
\text { proteína p53 }\end{array}$ & $\begin{array}{c}\text { Recidiva/progressão } \\
\text { n (\%) }\end{array}$ & Sem recidiva & Total \\
\hline Positiva & $6(30)$ & $\mathbf{n}(\%)$ & $\mathbf{n}(\%)$ \\
Negativa & & $4(20)$ & $10(50)$ \\
Total & $6(30)$ & $10(50)$ & $10(50)$ \\
\hline
\end{tabular}

O padrão de marcação imuno-histoquímica do tipo difuso (80\%) e a intensidade forte $(70 \%)$ foram as formas de expressão da presença da proteina p53 mais observadas.

Entretanto, o teste de Fisher não foi significativo em relação à intensidade $(p=0,5)$ e nem ao tipo de marcação imuno-histoquímica $(p=0,66)$, pois nas pacientes sem recidiva/progressão o padrão da imunoexpressão foi semelhante aos casos com recidiva/progressão, principalmente no que se refere ao tipo difuso de marcação.

A análise da presença da proteína p53 nos casos de recidiva/progressão foi realizada em um total de oito blocos parafinados, pelo fato de duas das seis pacientes com recidiva/progressão terem apresentado dois episódios de recidiva.

A proteína p53 mostrou-se presente na maioria dos casos de recidiva/progressão (75\%). Em apenas dois casos $(25 \%)$ a marcação imunohistoquímica para a proteína p53 revelou-se negativa. Na maioria dos casos de recidiva/progressão, o padrão de marcação imuno-histoquímica foi do tipo difuso $(83,3 \%)$ com intensidade forte
(83,3\%), à semelhança do verificado na lesão primária (ver Figuras 1B e 2 - lesão inicial e recorrência da mesma paciente). A única situação com marcação do tipo focal e intensidade fraca foi observada no caso de progressão da lesão.

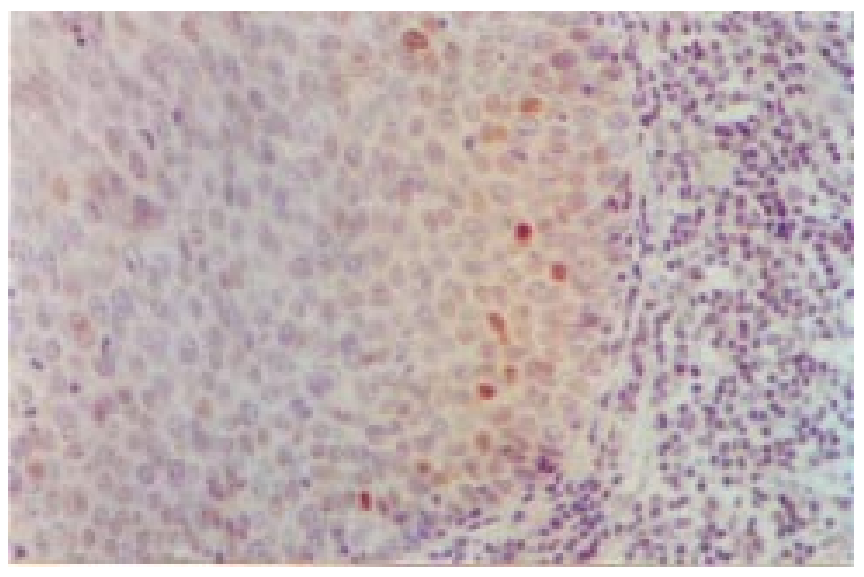

Figura 2 (X400) - Recidiva da lesão de VIN III 24 meses após o tratamento - Padrão de marcação imunohistoquímica para a proteína p53 do tipo difuso com intensidade forte, semelhante ao observado na lesão primária. 


\section{Discussão}

Diferentes estudos mostram índices de progressão da VIN III em pacientes tratadas que variam de 2 a 10\% $\%^{1,7,8,11-13}$. Quanto à progressão da VIN III em pacientes não tratadas, Jones e Rowan ${ }^{1}$ observaram que $87,5 \%$ das mulheres evoluíram para lesão invasora, independentemente da idade.

Em nossa série, todas as pacientes com VIN III foram tratadas cirurgicamente (excisão total da lesão). O percentual de progressão da lesão para carcinoma invasor foi de $5 \%(1 / 20)$.

Estabelecendo-se comparação entre as três áreas do trato genital inferior, vulva, vagina e colo, a vulva é a que mais contribui com os casos de recidiva das neoplasias intra-epiteliais ${ }^{9}$. Em nosso estudo, verificou-se recidiva em cinco de 20 pacientes submetidas a tratamento cirúrgico, correspondendo a $25 \%$ dos casos. Resultados semelhantes foram obtidos por Jones e Rowan ${ }^{1}$, Junge et al. ${ }^{9}$, Almeida Filho ${ }^{14}$ e Verdiani et al. ${ }^{15}$.

Os resultados revelam pequena tendência em direção à recidiva nos casos de distribuição multifocal das lesões. Todavia, tanto em nosso estudo quanto no de Junge et al. ${ }^{9}$, não houve diferença estatística entre os índices de recorrência e a localização uni ou multifocal da lesão primária, quando comparados ao grupo que não experimentou recidiva/progressão.

Dentre as seis pacientes (30\%) com recidiva/progressão avaliadas em nosso estudo, três apresentaram um único episódio de recorrência, duas experimentaram recidiva dupla e uma progrediu para carcinoma escamoso. Interessante notar que, do total de oito casos analisados, sete $(87,5 \%)$ recidivaram na mesma localização da lesão vulvar primária. Em apenas um caso a recidiva ocorreu em local diferente da lesão inicial. É importante frisar que as pacientes que apresentaram distribuição multifocal da lesão, na doença inicial, recidivaram com lesão única ou múltipla, porém sempre respeitando os locais previamente comprometidos. Da mesma forma, no único caso de progressão o terço posterior esteve comprometido tanto na doença inicial quanto na evolução para carcinoma. Isto confirma os achados de Junge et al. ${ }^{9}$ de que as recidivas de VIN III tendem a ocorrer na mesma área da doença inicial.

$\mathrm{Na}$ literatura consultada o tempo médio para ocorrência de recidiva da lesão é variável. Poucos são os trabalhos que falam sobre este período. Segundo Junge et al. ${ }^{9}$ o tempo médio entre a lesão inicial e a primeira recorrência situa-se em torno de 30 meses. Para Herod et al. ${ }^{8}$ os casos de recidiva tendem a ocorrer nos primeiros quatro anos que se seguem ao tratamento.
Em nosso estudo, o tempo médio entre a lesão primária e a primeira recidiva/progressão da lesão foi de 24,5 meses. A maioria das recidivas ocorreu em período superior a 12 meses. O único caso de progressão para carcinoma escamoso aconteceu em periodo inferior a um ano. O tempo de acompanhamento das pacientes com VIN III tratadas é fundamental para a avaliação da ocorrência de recidiva/progressão. É aconselhável seguimento mais prolongado dessas pacientes, pois sabe-se da ocorrência de progressão para carcinoma, após tratamento da VIN III, em períodos superiores a quatro anos ${ }^{13}$.

Em nossa série, 15 pacientes, nove do grupo sem e seis do grupo com recidiva/progressão (75\%), foram seguidas por período superior a 12 meses. Em cinco mulheres (25\%) pertencentes ao grupo que não apresentou recidiva ou progressão o seguimento foi realizado por período inferior a um ano, pois entraram em nossa pesquisa sete meses antes de seu término. Observamos diferença significativa quando estabelecemos comparação entre os dois grupos, em relação ao tempo médio de seguimento. Como o tempo médio de recidiva/ progressão verificado em nossa série foi de 24,5 meses, os dados sugerem que talvez observemos maior incidência de recorrência, ou até mesmo de progressão da lesão, caso estas pacientes sejam acompanhadas por período de tempo mais prolongado.

Raras são as publicações sobre a presença da proteína p53 nas VIN III. Até o presente momento, não encontramos estudos sobre o comportamento da proteína p53 nos casos de recidiva/ progressão da VIN III.

A inativação da proteína codificada pelo gene supressor de tumor P53, seja por mutação ou por degradação promovida pela proteína E6 dos HPV oncogênicos, parece ser fator importante no processo da carcinogênese vulvar ${ }^{10,16-19}$.

Embora, à semelhança de outros tipos de cânceres, a gênese do câncer de vulva possa ocorrer por mecanismos outros, independentes da inativação da proteína p53, a literatura, apesar de escassa, mostra taxas revelando a presença da proteína entre 10 e $50 \%$ dos casos de VIN III ${ }^{10,18,19}$.

Os estudos revelam que apenas os casos nos quais se detecta a presença da proteína p53 igual ou superior a 100 células positivas para 1.100 negativas (maior ou igual a $10 \%$ ) são considerados positivos pois, freqüentemente, refletem mutação do tipo missense entre os exons 5-8 do gene $P 53^{20}$. A ausência da proteína p53 pode, simplesmente, indicar presença do tipo selvagem, da proteína, traduzindo ausência de mutação no gene P53; mutações outras, que não as do tipo missense; degradação da p53, induzida por ligação com pro- 
teínas virais (E6 dos HPV oncogênicos) ou deleção do gene $P 53^{16,20}$.

Em nossa série, a presença da proteína p53 foi observada nas cinco lesões primárias que cursaram com recidiva e na que evoluiu para carcinoma escamoso. Todavia, detectou-se a presença da proteína p53 em quatro pacientes não pertencentes ao grupo que experimentou recidiva/progressão. Como essas mulheres foram as últimas a entrar em nossa pesquisa, o tempo de seguimento foi de apenas seis meses. Considerandose que nosso tempo médio de recorrência foi de 24 meses, é possivel que não tenha havido tempo suficiente para o surgimento de recidiva/progressão. Por outro lado, não se verificou presença da proteína p53 nos casos em que não houve recidiva/progressão da doença. Desta forma, tudo leva a crer que mutação no gene P53 apresenta intima relação com o aparecimento das VIN III e com sua progressão para carcinoma.

O padrão de marcação imuno-histoquímica predominante na lesão primária foi o tipo difuso $(80 \%)$ com intensidade forte $(70 \%)$. Segundo Stenmark-Askmalm et al. ${ }^{6}$, grande acúmulo nuclear da proteína p53, com conseqüente padrão de marcação imuno-histoquímica do tipo forte, indica importante instabilidade genética com rápida proliferação tumoral. Apenas uma paciente, em nossa série, apresentou marcação imunohistoquímica com combinação difusa/fraca. Segundo Esrig et al. ${ }^{21}$, a heterogeneidade na intensidade ou no tipo de marcação deve-se ao ponto de mutação no gene $P 53$ ou à combinação do tipo mutado com o selvagem da p53.

A presença da proteína p53 foi observada na maioria dos casos de recidiva/ progressão (75\%). Em apenas dois casos (25\%) ela esteve ausente. O padrão de marcação imuno-histoquímica foi constituído, predominantemente, pelo tipo difuso $(83,3 \%)$ com intensidade forte $(83,3 \%)$. Esse padrão foi semelhante ao da lesão primária. O único caso $(16,7 \%)$ que apresentou marcação imuno-histoquímica do tipo focal com intensidade fraca refere-se ao da progressão da lesão para carcinoma. Interessante notar que esse padrão de marcação focal/fraco também foi encontrado na doença inicial.

Baseado nos resultados obtidos em nossa pesquisa, verifica-se que as recidivas/progressão das VIN III tendem a ocorrer na mesma área da doença primária, indicando a presença de campo molecular alterado preexistente às alterações morfológicas. Da mesma forma, a presença de mutação no gene $P 53$, com conseqüente perda de função da proteína p53, parece estar associada ao maior risco de recidiva ou progressão das VIN III.

A partir do observado, esperamos que estudos futuros de biologia molecular associados à análise das margens cirúrgicas das VIN III funci- onem como indicadores de recidiva ou de progressão dessas lesões.

\section{SUMMARY}

Purpose: to evaluate p53 overexpression value in vulvar intraepithelial neoplasia (VIN) III recurrence/progression. Methods: twenty patients with undifferentiated VIN III were selected and followed up every six months for four years and divided into two groups: fourteen without and six with recurrence/progression lesion. The recurrence/progression cases were distributed as follows: in three patients recurrence occurred only once; in two, twice, and only one progressed to squamous cancer. In both groups the site of vulvar lesion and p53 overexpression and immunostaining pattern were analyzed. A similar study was performed in recurrencel progression cases, besides the analysis of the time interval to occur the arise of recurrence/progression.

Results: recurrence was observed in $25 \%$ of the cases and, in $5 \%$, progression to carcinoma. The mean time interval for recurrence was 24.5 months. Multifocal location of the initial lesion was the predominant form (50\%) in both groups. In the majority of the cases (87.5\%) recurrence/progression occurred at the same site of the initial vulvar lesion. p53 overexpression was observed in $50 \%$ of the VIN III primary lesions and in $75 \%$ of the recurrence/progression cases.

Conclusions: $p 53$ overexpression seems to play an important role in VIN III pathogenesis and may predict the clinical course of the lesions. VIN III recurrence/progression has a tendency to occur in the same area of the initial lesion, suggesting the presence of molecular disturbance.

KEY WORDS: Vulvar intraepithelial neoplasia. Vulva: preneoplastic lesions. Oncogenes.

\section{Referências}

1. Jones RW, Rowan DM. Vulvar intraepithelial neoplasia III: a clinical study of the outcome in 113 cases with relation to the later development of invasive vulvar carcinoma. Obstet Gynecol 1994; 84:741-5.

2. Lin MC, Mutter GL, Trivijisilp P, Boynton KA, Sun D, Crum CP. Patterns of allelic loss (LHO) in vulvar squamous carcinomas and adjacent noninvasive epithelia. Am J Pathol 1998; 152:1313-8.

3. Flowers LC, Wistuba II, Scurry J, et al. Genetic changes during the multistage pathogenesis of human papillomavirus positive and negative vulvar carcinomas. J Soc Gynecol Invest 1999; 6:213-21.

4. Sionov RV, Haupt Y. The cellular response to p53: the decision between life and death. Oncogene $1999 ; 18: 6145-57$. 
5. Ellisen LW, Haber DA. Hereditary breast cancer. Annu Rev Med 1998; 49:425-36.

6. Stenmark-Askmalm M, Stal O, Olsen K, Nordenskjold B. p53 as a prognostic factor in stage I breast cancer. Br J Cancer 1995; 72:715-9.

7. Fiorica JV, Cavanagh D, Marsden DE, Shepherd JH, Ruffolo EH, Songster CL. Carcinoma in situ of the vulva: 24 years' experience in southwest Florida. South Med J 1988; 81:589-93.

8. Herod JJ, Shafi MI, Rollason TP, Jordan JA, Luesley DM. Vulvar intraepithelial neoplasia: long term follow up of treated and untreated women. Br J Obstet Gynaecol 1996; 103:446-52.

9. Junge J, Poulsen H, Horn T, Hording U, Lundvall F. Prognosis of vulvar dysplasia and carcinoma in situ with special reference to histology and types of human papillomavirus (HPV). APMIS 1997; 105:963-71.

10.Emanuels AG, Koudstaal J, Burger MP, Hollema H. In squamous cell carcinoma of the vulva, overexpression of p53 is a late event and neither p53 nor $\mathrm{mdm}^{2}$ expression is a useful marker to predict lymph node metastases. Br J Cancer 1999; 80:38-43.

11.Barbero M, Michelleti L, Preti M, et al. Biologic behavior of vulvar intraepithelial neoplasia. Histologic and clinical parameters. J Reprod Med 1993; 38:108-12.

12.Buscema J, Naghashfar Z, Sawada E, Daniel R, Woodruff JD, Shah K. The predominance of human papillomavirus type 16 in vulvar neoplasia. Obstet Gynecol 1988; 71:601-6.

13.Küppers V, Stiller M, Somville T, Bender HG. Risk factors for recurrent VIN. Role of multifocality and grade of disease. J Reprod Med 1997; 42:140-4.
14.Almeida Filho GL. Neoplasia intra-epitelial vulvar: estudo clínico e histopatológico [tese]. Rio de Janeiro: Universidade Federal do Rio de Janeiro; 1998.

15.Verdiani LA, Juliato CRT, Derchain SFM, Ferro, JE. Neoplasias intra-epitelial vulvar: análise clinicopatológica. Rev Bras Ginecol Obstet 1998; 20:371-6.

16.Kagie MJ, Kenter GG, Tollenaar RA, Hermans J, Trimbos JB, Fleuren GJ. p53 protein overexpression is common and independent of human papillomavirus infection in squamous cell carcinoma of the vulva. Cancer 1997; 80:1228-33.

17.Lee YY, Wilczynski SP, Chumakov A, Chih D, Koeffler HP. Carcinoma of the vulva: HPV and p53 mutations. Oncogene 1994; 9:1655-9.

18.McConnell DT, Miller ID, Parkin DE, Murray GI. p53 protein expression in a population-based series of primary vulval squamous cell carcinoma and immediate adjacent field change. Gynecol Oncol $1997 ; 67: 248-54$.

19.Milde-Langosch K, Albrecht K, Joram S, Schlechte $\mathrm{H}$, Giessing $\mathrm{M}$, Loning $\mathrm{T}$. Presence and persistence of the HPV infection and p53 mutation in cancer of the cervix uteri and the vulva. Int J Cancer 1995; 63:639-45.

20.Weller M. Predicting response to cancer chemotherapy: the role of p53. Cell Tissue Res 1998; 292:435-45.

21.Esrig D, Spruck CH 3rd, Nichols PW, et al. p53 nuclear protein accumulation correlates with mutations in the p53 gene, tumor grade, and stage in bladder cancer. Am J Pathol 1993; 143:1389-97.

Recebido em: $7 / 8 / 2001$ Aceito com modificações em: 26/11/2001 